\title{
47 Übergewicht und Krankheitsrisiko
}

\author{
(c) Springer-Verlag GmbH Deutschland, ein Teil von Springer Nature 2018 \\ D. Mathias, Fit und gesund von 1 bis Hundert \\ https://doi.org/10.1007/978-3-662-56307-6_47
}

Der Überlebensvorteil in früheren Zeiten, große Fettreserven im Körper anlegen zu können, wandelt sich in unserer heutigen Gesellschaft mit ihren fast unbegrenzten Nahrungsressourcen in sein Gegenteil. Übergewicht und Adipositas sind Ursachen von etwa 60 sehr ernsten Folgekrankheiten. $\mathrm{Zu}$ ihnen gehören u. a. die Altersdemenz ( $\triangleright$ Kap. 43), Krebs (Kyrgiou et al. 2017), Bluthochdruck, Diabetes, chronische Niereninsuffizienz sowie ein gehäuftes Auftreten arteriosklerotischer Komplikationen wie Herzinfarkt und Schlaganfall (Lu et al. 2014; Chang et al. 2016; Lauby-Secretan et al. 2016; O’Donnel et a. 2016; Campbell et al. 2016; Lassale et al. 2017). Dabei steigt das Risiko speziell für koronare Herzkrankheiten nach den Studiendaten von 302.296 Personen in Europa und den USA mit Beobachtungszeiten von 6-35 Jahren um $17 \%$, wenn der BMI zwischen 25 und 29,9 liegt und um $45 \%$ bei einem BMI über 30. Diese Angaben wurden bereits um die Risiken erhöhter Blutdrucke und Cholesterinwerte bereinigt (Bogers et al. 2007).

Beim gleichzeitigen Vorliegen von kardiovaskulären Erkrankungen und Diabetes ist das Todesfallrisiko in etwa verdoppelt. Dieser Zusammenhang zeigte sich nach Auswertung einer Metaanalyse aus 102 prospektiven Studien mit knapp 700.000 Patienten (ERFC 2010).

Ergebnisse der Nurses' Health Study betonen die Bedeutung des Erhalts eines normalen Körpergewichts schon vom frühen Erwachsenenalter an. So schränkt bei Frauen ein starkes Übergewicht im Alter von 50 Jahren die Aussicht auf einen guten Gesundheitszustand im späteren Alter von 70 Jah-

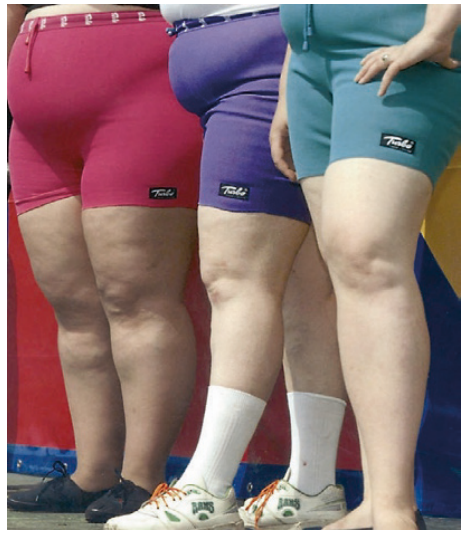

- Abb. $47.1 \odot \mathrm{dpa} / \mathrm{akg}$

ren drastisch ein (Sun et al. 2009). Beginnt die Übergewichtigkeit bereits mit 18 Jahren, verringern sich die Chancen, bis ins hohe Alter gesund und rüstig zu bleiben, noch mehr. Jedes Kilo Gewichtszunahme nach dem 18. Lebensjahr mindert diese Chancen um ca. $5 \%$.

Eine Besonderheit wird immer wieder in größeren Studien beschrieben. Leiden nämlich Personen bereits an Herzkrankheiten, chronischem Nierenversagen, Diabetes oder Krebs, dann haben die Patienten mit einem leicht erhöhten BMI oft bessere Überlebenschancen als schlanke Patienten (Carnethon et al. 2012; Sharma et al. 2014). Dieses Phänomen wird in der Literatur als AdipositasParadoxon bezeichnet (Angeras et al. 2013; de Schutter et al. 2014). Es findet sich meist dann, wenn eine vermehrte Muskelmasse die Erhöhung des BMI bewirkt (Srikanthan et al. 2016). 